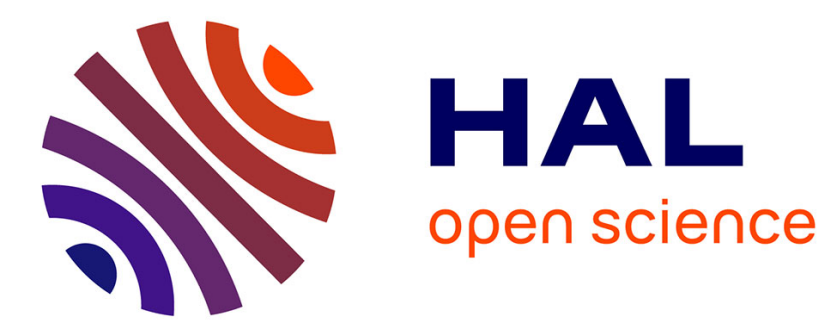

\title{
Remarkable polyhedra related to set functions, games and capacities
}

\author{
Michel Grabisch
}

\section{To cite this version:}

Michel Grabisch. Remarkable polyhedra related to set functions, games and capacities. TOP, 2016, 24 (2), pp.301-326. 10.1007/s11750-016-0421-4 . hal-01372858

\section{HAL Id: hal-01372858 \\ https://hal.science/hal-01372858}

Submitted on 27 Sep 2016

HAL is a multi-disciplinary open access archive for the deposit and dissemination of scientific research documents, whether they are published or not. The documents may come from teaching and research institutions in France or abroad, or from public or private research centers.
L'archive ouverte pluridisciplinaire HAL, est destinée au dépôt et à la diffusion de documents scientifiques de niveau recherche, publiés ou non, émanant des établissements d'enseignement et de recherche français ou étrangers, des laboratoires publics ou privés. 


\title{
Remarkable polyhedra related to set functions, games and capacities
}

\author{
Michel GRABISCH \\ Paris School of Economics, University of Paris I \\ 106-112, Bd de l'Hôpital, 75013 Paris, France \\ michel.grabisch@univ-paris1.fr
}

\begin{abstract}
Set functions are widely used in many domains of Operations Research (cooperative game theory, decision under risk and uncertainty, combinatorial optimization) under different names (TU-game, capacity, nonadditive measure, pseudo-Boolean function, etc.). Remarkable families of set functions form polyhedra, e.g., the polytope of capacities, the polytope of $p$-additive capacities, the cone of supermodular games, etc. Also, the core of a set function, defined as the set of additive set functions dominating that set function, is a polyhedron which is of fundamental importance in game theory, decicion making and combinatorial optimization. This survey paper gives an overview of these notions and studies all these polyhedra.
\end{abstract}

Keywords: TU-game, capacity, nonadditive measure, pseudo-Boolean function, Möbius transform, supermodular game, $p$-additive game, multichoice game, core.

\section{Introduction}

Set functions, that is, functions assigning a real number to any subset of a universal set, are encountered in many domains of Operations Research and discrete mathematics, often under different names. Let us give some examples. In (cooperative) game theory, the characteristic function of a game with transferable utility is a set function vanishing on the empty set. They are often abbreviated as TU-games, or simply games. In decision under risk or uncertainty, recent nonadditive models use generalizations of probability measures which are called capacities (Choquet [6]), or nonadditive measures (Denneberg [9]), fuzzy measures (Sugeno [50]), monotone measures, etc. These are set functions vanishing on the empty set (hence games) and monotone w.r.t. set inclusion. Pseudo-Boolean functions, introduced by Hammer and Rudeanu [32], are real-valued mappings whose domain is the set of Boolean vectors, and are therefore equivalent to set functions. They have many applications in computer sciences, graph theory, data mining, etc. (see, e.g., Crama and Hammer [7, Ch. 13], and O'Donnell [41]). In combinatorial optimization, submodular and monotone set functions are considered, since they appear as the rank function of a matroid. They are studied at length in the monograph of Fujishige [18].

Many of the above cited domains are related to optimization problems, linear programming and polyhedra. One of the reasons is that remarkable families of set functions are defined by linear inequalities, and therefore form polyhedra. This is the case for capacities (monotone normalized set functions), supermodular games, or $p$-additive games, defined through their Möbius transform. Another reason is that the core of a set function is a fundamental concept in most of the above cited domains. It is the set of additive set functions dominating a given 
set function, and is therefore a polyhedron. In game theory, it is one of the most popular solution concept. In decision theory, it gives the set of compatible probability measures. In combinatorial optimization, it is known as the base polyhedron associated to a polymatroid.

The aim of the paper is to make a survey of these aspects, summarizing most relevant classical results, showing recent ones, and exploring some generalizations like games with restricted domain, multichoice games, the $k$-additive core, etc. It also aims at enhancing the close connections between cooperative game theory, combinatorial optimization, decision theory and Operations Research in general. Indeed, as it will be seen, many results have been proved at the same time in different domains. Many other aspects of set functions, games and capacities can be found in the monograph of the author [24].

The paper is organized as follows. Section 2 gives the necessary background on games, capacities, pseudo-Boolean functions, posets and lattices, the Möbius transform, multichoice games and $k$-ary capacities. Sections 3 to 6 study the polyhedra formed by various families of set functions (capacities, $p$-additive capacities, $k$-ary capacities, and supermodular games). The core of games is studied in detail in Section 7, for classical games and for games with a restricted domain. Finally, the $k$-additive core is studied in Section 8.

\section{Background}

Throughout the paper, we consider a finite set $N$ with $|N|=n$. We often omit braces for singletons and sets, as far as no ambiguity menaces, writing, e.g., $S \cup i$ instead of $S \cup\{i\}, 12$ instead of $\{1,2\}$, etc.

Basic definitions: set functions, games and capacities. A set function on $N$ is a mapping $\xi: 2^{N} \rightarrow \mathbb{R}$ assigning to every subset $S \subseteq N$ a real number. A game on $N$ is a set function $v$ vanishing on the empty set: $v(\varnothing)=0$. We say that a game is monotone if $S \subseteq T \subseteq N$ implies $v(S) \leq v(T)$, and normalized if $v(N)=1$. In decision theory, a monotone normalized game is called a capacity ${ }^{1}$ (Choquet [6]), which we denote by $\mu .^{2}$ A 0 -1-capacity (a.k.a. simple game) is a capacity taking value on $\{0,1\}$. A zero-normalized game $v$ is a game such that $v(\{i\})=0$ for every $i \in N$. We denote the set of capacities on $N$ by $\mathrm{C}(N)$, and the set of games on $N$ by $\mathcal{G}(N)$.

The conjugate of a game $v$ is a game $\bar{v}$ defined by

$$
\bar{v}(S)=v(N)-v(N \backslash S) \quad\left(S \in 2^{N}\right) .
$$

A game is said to be:

(i) additive if for every disjoint $S, T$ in $2^{N}$, we have

$$
v(S \cup T)=v(S)+v(T) ;
$$

(ii) supermodular if for every $S, T \in 2^{N}$, we have

$$
v(S \cup T)+v(S \cap T) \geq v(S)+v(T) ;
$$

(iii) submodular if the reverse inequality holds.

\footnotetext{
${ }^{1}$ In general, normalization is not required. We assume it throughout this paper for convenience.

${ }^{2}$ As far as possible, we stick to these notations: $\xi$ for set functions, $v$ for games and $\mu$ for capacities.
} 
Instances in Operations Research. In cooperative game theory, $N$ represents the set of players, and the function $v$ (whose exact name is characteristic function of a game with transferable utility) gives for each coalition $S \subseteq N$ of players its worth $v(S)$, defined as the maximal benefit or cost saving achievable by cooperation of the members of $S$.

In decision theory, $N$ is typically the set of states of nature, subsets of $N$ are called events, and $\mu(S)$ quantifies the uncertainty pertaining to the realization of $S$.

Particular set functions arise also in combinatorial optimization and Operations Research. In combinatorial optimization, a pair $(N, \rho)$ where $\rho$ is a submodular capacity, is called a polymatroid (see, e.g., Fujishige [18]). This refers to the rank function of a matroid, which is always submodular. In reliability theory, set functions model the structure of a multicomponent system and permit to study its lifetime: given a $n$-component system and supposing that each component can be in two states (0: failure, 1: functioning), the state of the whole system is ruled by a function $\phi:\{0,1\}^{n} \rightarrow\{0,1\}$. It is most often assumed that $\phi$ is nondecreasing in each place, hence $\phi$ is nothing other than a 0-1-capacity (or simple game).

Pseudo-Boolean functions. Set functions can also be seen as pseudo-Boolean functions, a widely developed area in Operations Research, after the seminal work of Hammer and Rudeanu [32] (see Crama and Hammer [7, Ch. 13]). A pseudo-Boolean function is a function $f:\{0,1\}^{n} \rightarrow$ $\mathbb{R}$; they are equivalent to set functions through the isomorphism $\{0,1\}^{n} \equiv 2^{N}$, where a set $S$ is represented by its characteristic function $1_{S}$ :

$$
f \leftrightarrow \xi \quad f\left(1_{S}\right) \leftrightarrow \xi(S) .
$$

It is easy to check that any pseudo-Boolean function can be put under the form:

$$
f(x)=\sum_{T \subseteq N} f\left(1_{T}\right) \prod_{i \in T} x_{i} \prod_{i \in T^{c}}\left(1-x_{i}\right) \quad\left(x \in\{0,1\}^{n}\right) .
$$

Rearranging the terms so as to obtain a sum of monomials, we get:

$$
f(x)=\sum_{T \subseteq N} a_{T} \prod_{i \in T} x_{i} \quad\left(x \in\{0,1\}^{n}\right) .
$$

The degree of a pseudo-Boolean function is the highest degree of the monomials in (3), where the degree of the monomial $\prod_{i \in T} x_{i}$ is simply $|T|$.

Posets and lattices. A poset (partially ordered set) $(P, \preceq)$ is a set $P$ endowed with a partial order $\preceq$ (reflexive, antisymmetric and transitive binary relation). If $x \prec y$ and there is no $z$ such that $x \prec z \prec y$, we write $x \prec \cdot y$ and say that $x$ is a predecessor of $y$. For any two elements $x, y$ in a poset $(P, \preceq)$ such that $x \preceq y$, the interval $[x, y]$ is defined by $[x, y]=\{z \in$ $P \mid x \preceq z \preceq y\}$. A lattice $(L, \preceq)$ is a poset satisfying the following property: for every $x, y \in L$, their infimum (greatest lower bound) $x \wedge y$ and supremum (least upper bound) $x \vee y$ exist. A lattice is distributive if $\vee, \wedge$ obey distributivity, i.e., $x \vee(y \wedge z)=(x \vee y) \wedge(x \vee z)$ (equivalently, $x \wedge(y \vee z)=(x \wedge y) \vee(x \wedge z))$.

In a lattice $(L, \preceq)$, an element $x \in L$ is said to be join-irreducible if it is not the least element of $L$ and it cannot be written as a supremum of other elements. In a finite lattice, this is equivalent to say that $x$ has only one predecessor. We denote by $\mathcal{J}(L, \preceq)$ or $\mathcal{J}(L)$ if $\preceq$ is clear from the context the set of join-irreducible elements of $L$.

$Q \subseteq P$ is a downset of a poset $(P, \preceq)$ if $x \in Q$ and $y \preceq x$ imply $y \in Q$. The set of all downsets of a poset $(P, \preceq)$ is denoted by $\mathcal{O}(P, \preceq)$ or simply $\mathcal{O}(P)$. An important result of 
Birkhoff [4] says that a lattice $(L, \preceq)$ is distributive if and only if it is isomorphic to the lattice $(\mathcal{O}(\mathcal{J}(L)), \subseteq)$.

A chain in a poset $(P, \preceq)$ is a sequence $x_{1}, \ldots, x_{q}$ of distinct elements in $P$ such that $x_{1} \prec \cdots \prec x_{q}$. A chain is maximal if no supersequence is a chain. Two elements $x, y \in(P, \preceq)$ are incomparable if none of $x \preceq y$ or $y \preceq x$ holds. An antichain is a set of elements in $P$ which are pairwise incomparable.

We illustrate the previous notions and result with $N=\{1, \ldots, n\}$ the set of players. The set $2^{N}$ endowed with $\subseteq$ is a lattice, called a Boolean lattice. Any subcollection $\mathcal{F}$ of $2^{N}$ can be seen as a poset when endowed with $\subseteq \mathcal{F}$ can be a lattice, but in this case, infimum and supremum are not necessarily intersection and union. For instance, take $n=3, \mathcal{F}=\{\varnothing, 1,2,3,123\}$. Then $1 \vee 2=123 \neq 1 \cup 2$. If $(\mathcal{F}, \subseteq)$ is a distributive lattice, then Birkhoff's result amounts to saying that there exists a partial order $\preceq$ on $N$ generating $\mathcal{F}$, by taking all downsets of $(N, \preceq)$ and ordering them by inclusion. The converse being true as well, there is a bijection between partial orders on $N$ and collections $\mathcal{F}$ that are distributive lattices. It is to be noted that with this construction, the supremum and infimum in $\mathcal{F}$ are union and intersection. This is illustrated in Figure 1.
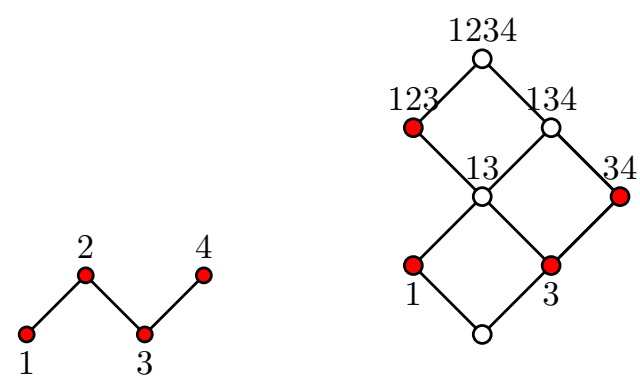

Figure 1: $n=4$. Left: a poset $(N, \preceq)$. Right: the set of downsets of $(N, \preceq)$, ordered by inclusion. It is a distributive lattice $(\mathcal{F}, \subseteq)$. Its join-irreducible elements are in red, and the poset of join-irreducible elements $(\mathcal{J}(\mathcal{F}), \subseteq)$ is isomorphic to $(N, \preceq)$

The Möbius transform. (Rota [44]) Let us consider a poset $(P, \preceq)$, and suppose that it is locally finite (i.e., all intervals $[x, y]$ are finite), and has a least element 0 . Consider the following system of equations, where $f$ is given:

$$
f(x)=\sum_{y \preceq x} g(y) \quad(x \in P) .
$$

There is always a unique solution to (4), i.e., it is always possible to express $g$ in terms of $f$. Then $g$ is called the Möbius inverse of $f$, and is given by

$$
g(x)=\sum_{y \preceq x} \mu(y, x) f(y)
$$

where $\mu$, called the Möbius function, is defined inductively by

$$
\mu(x, y)= \begin{cases}1, & \text { if } x=y \\ -\sum_{x \preceq t \prec y} \mu(x, t), & \text { if } x \prec y \\ 0, & \text { otherwise. }\end{cases}
$$

We apply this general theory to set functions, that is, the poset under consideration is $\left(2^{N}, \subseteq\right)$. We find that to any set function $\xi$ on $N$ we can associate its Möbius inverse $m^{\xi}$ : 
$2^{N} \rightarrow \mathbb{R}$, unique solution of the linear system:

$$
\xi(S)=\sum_{T \subseteq S} m^{\xi}(T) \quad\left(S \in 2^{N}\right)
$$

and explicitely given by

$$
m^{v}(S)=\sum_{T \subseteq S}(-1)^{|S \backslash T|} v(T) \quad\left(S \in 2^{N}\right) .
$$

Considering the mapping $\xi \mapsto m^{\xi}$ on $\mathbb{R}^{2^{N}}$ as a transform over the set of set functions, we call $m^{\xi}$ the Möbius transform of $\xi$. In game theory, the Möbius transform of a game is known as the Harsanyi dividends (Harsanyi [33]). Comparing (7) with (3), one can see that they are the same up to the isomorphism $f \leftrightarrow \xi$, from which we conclude that the coefficients $a_{T}$ are nothing other than the Möbius transform of $\xi$ (or $f$ ).

Unanimity games. Given $\varnothing \neq T \subseteq N$, the unanimity game centered on $T$ is a game defined by:

$$
u_{T}(S)= \begin{cases}1, & \text { if } S \supseteq T \\ 0, & \text { otherwise. }\end{cases}
$$

It is well known that the set of unanimity games constitutes a basis of the $\left(2^{n}-1\right)$-dim vector space of games. Adding the set function $u_{\varnothing}$ defined by $u_{\varnothing}(S)=1$ for every $S \in 2^{N}$, we get a basis for the $2^{n}$-dim vector space of set functions. The expression of any set function in this basis is therefore:

$$
\xi=\sum_{T \subseteq N} a_{T} u_{T}
$$

which yields

$$
\xi(S)=\sum_{T \subseteq N} a_{T} u_{T}(S)=\sum_{T \subseteq S} a_{T}
$$

from which it becomes obvious that the coordinates $a_{T}$ of a set function in this basis are simply the Möbius transform of $\xi$ (see (7)).

$p$-additive games and capacities. A game (or a capacity) $v$ on $N$ is (at most) p-additive $(p \in\{1, \ldots, n\})$ if its Möbius transform satisfies

$$
m^{v}(S)=0 \text { if }|S|>p .
$$

1-additive games are ordinary additive games. $p$-additive games correspond to pseudo-Boolean functions whose polynomial representation is of degree at most $p$. Clearly, $p$-additive games with $p<n$ require less coefficients to be defined, precisely at most

$$
\kappa(p)=\left(\begin{array}{l}
n \\
1
\end{array}\right)+\left(\begin{array}{l}
n \\
2
\end{array}\right)+\cdots+\left(\begin{array}{l}
n \\
p
\end{array}\right) .
$$

The set of at most $p$-additive capacities is denoted by $\mathrm{e} \leq p(N)$. 
Multichoice games and $k$-ary capacities. Identifying $2^{N}$ with $\{0,1\}^{N}$ and considering any game $v$, the quantity $v(S)$ can be rewritten as $v\left(1_{S}\right)$, replacing coalitions by profiles of participation (0: do not participate, 1: participate). The idea of multichoice games (Hsiao and Raghavan [34]) is to consider several levels of participation: $0,1, \ldots, k$. Specifically, a multichoice game or $k$-choice game is a mapping $v:\{0,1, \ldots, k\}^{N} \rightarrow \mathbb{R}$ such that $v(0, \ldots, 0)=0$, which assigns to any participation profile $z$ a number $v(z)$. A multichoice game is monotone if $z \leq z^{\prime}$ (coordinatewise) implies $v(z) \leq v\left(z^{\prime}\right)$, and normalized if $v(k, \ldots, k)=1$. With $k=1$, classical games are recovered. Monotone normalized $k$-multichoice games are called $k$ ary capacities (Grabisch and Labreuche [25]). The set of $k$-ary capacities is denoted by $\mathcal{C}_{k}(N)$.

The Möbius transform $m^{v}$ of a multichoice game $v$ can be obtained from the general formula (4), which becomes in this case

$$
v(z)=\sum_{y \leq z} m^{v}(y) \quad\left(z \in\{0, \ldots, k\}^{N}\right) .
$$

The unique solution of this system is

$$
m^{v}(z)=\sum_{y \leq z: z_{i}-y_{i} \leq 1 \forall i \in N}(-1)^{\sum_{i \in N}\left(z_{i}-y_{i}\right)} v(y) \quad\left(z \in\{0, \ldots, k\}^{N}\right) .
$$

Based on this definition, a multichoice game is (at most) $p$-additive if $m^{v}(z)=0$ for every profile $z$ whose support contains more than $p$ elements, i.e., $|\operatorname{supp}(z)|>p$, where

$$
\operatorname{supp}(z)=\left\{i \in N \mid z_{i}>0\right\} .
$$

The set of at most $p$-additive $k$-ary capacities is denoted by $\mathcal{C}_{k}^{\leq p}(N)$.

\section{The polytope of capacities}

The set $\mathrm{C}(N)$ of capacities (monotone normalized games) on $N$ being defined by a set of linear equalities, it is a convex closed polyhedron of dimension $2^{n}-2$, which is bounded. Facets of this polytope are the irredundant monotonicity conditions

$$
\mu(S \cup i)-\mu(S) \leq 0 \quad(i \in N, S \subseteq N \backslash i) .
$$

The extreme points (vertices) of this polytope have been determined by Stanley [48], and rediscovered by Radojevic [42].

Theorem 1. The set of extreme points of $\mathfrak{C}(N)$ is the set of 0 -1-capacities (simple games).

As is well known, simple games are determined through their collection of winning coalitions. A coalition $S$ is winning for a simple game $v$ if $v(S)=1$. By monotonicity, it follows that a simple game $v$ is entirely determined by the collection of its minimal (in the sense of inclusion) winning coalitions. By definition, if $S, T$ are two minimal winning coalitions, $S \nsubseteq T$ and $T \nsubseteq S$ hold, so that the collection of minimal winning coalitions forms an antichain in the lattice $\left(2^{N}, \subseteq\right)$. It is known that the number of antichains in $2^{N}$, or equivalently, the number of monotonic Boolean functions of $n$ variables is the Dedekind number $M(n)$. It follows that the number of vertices of the polytope of capacities on $N$ is $M(n)-2$ (because the antichain $\{\varnothing\}$, corresponding to the constant set function 1 , and the empty antichain, corresponding to the null set function, have to be removed).

There is no known closed-form formula for an exact computation of these numbers. Up to now, only the nine first numbers are known. 


\begin{tabular}{|r|r|}
\hline$n$ & $M(n)$ \\
\hline 0 & 2 \\
1 & 3 \\
2 & 6 \\
3 & 20 \\
4 & 168 \\
5 & 7581 \\
6 & 7828354 \\
7 & 2414682040998 \\
8 & 56130437228687557907788 \\
\hline
\end{tabular}

Table 1: The Dedekind numbers $M(n)$ for $0 \leq n \leq 8$

Observe that by Theorem 1, this polytope is inscribed into the unit hypercube $\{0,1\}^{2^{n}-2}$, and the main diagonal belongs to the polytope. It follows that the diameter of the polytope of capacities is the length of the diagonal, i.e., $\sqrt{2^{n}-2}$. An interesting question is: in which hypercube is inscribed the set of capacities when they are expressed through the Möbius transform, i.e., in the basis of unanimity games? The exact bounds of the Möbius transform of a capacity have been investigated by Grabisch and Miranda [29]. The result is surprising: the bounds grow exponentially fast with $n$.

Theorem 2. For any capacity $\mu$, its Möbius transform satisfies for any $A \subseteq N,|A|>1$ :

$$
-\left(\begin{array}{c}
|A|-1 \\
l_{|A|}^{\prime}
\end{array}\right) \leq m^{\mu}(A) \leq\left(\begin{array}{c}
|A|-1 \\
l_{|A|}
\end{array}\right)
$$

with

$$
l_{|A|}=2\left\lfloor\frac{|A|}{4}\right\rfloor, \quad l_{|A|}^{\prime}=2\left\lfloor\frac{|A|-1}{4}\right\rfloor+1
$$

and for $|A|=1<n$ :

$$
0 \leq m^{\mu}(A) \leq 1,
$$

and $m^{\mu}(A)=1$ if $|A|=n=1$. These upper and lower bounds are attained by the normalized capacities $\mu_{A}^{*}, \mu_{A *}$, respectively:

$$
\begin{aligned}
\mu_{A}^{*}(B) & = \begin{cases}1, & \text { if }|A|-l_{|A|} \leq|B \cap A| \\
0, & \text { otherwise }\end{cases} \\
\mu_{A *}(B) & = \begin{cases}1, & \text { if }|A|-l_{|A|}^{\prime} \leq|B \cap A| \\
0, & \text { otherwise }\end{cases}
\end{aligned}
$$

for any $B \subseteq N$.

Table 2 below gives the first values of the bounds.

Using the Stirling's approximation $\left(\begin{array}{c}2 n \\ n\end{array}\right) \simeq \frac{4^{n}}{\sqrt{\pi n}}$ for $n \rightarrow \infty$, we obtain the asymptotic behavior of the bounds:

$$
-\frac{4^{\frac{n}{2}}}{\sqrt{\frac{\pi n}{2}}} \leq m^{\mu}(N) \leq \frac{4^{\frac{n}{2}}}{\sqrt{\frac{\pi n}{2}}}
$$

The theorem directly gives the coordinates of the vertices of the $\left(2^{n}-2\right)$-dim smallest rectangle inscribing the polytope of capacities. Finding its diameter is however more difficult. 


\begin{tabular}{|l|rrrrrrr|}
\hline$|A|$ & 1 & 2 & 3 & 4 & 5 & 6 & 7 \\
\hline u.b. of $m^{\mu}(A)$ & 1 & 1 & 1 & 3 & 6 & 10 & 15 \\
\hline l.b. of $m^{\mu}(A)$ & $1(0)$ & -1 & -2 & -3 & -4 & -10 & -20 \\
\hline
\end{tabular}

\begin{tabular}{|l|rrrrr|}
\hline$|A|$ & 8 & 9 & 10 & 11 & 12 \\
\hline u.b. of $m^{\mu}(A)$ & 35 & 70 & 126 & 210 & 462 \\
\hline l.b. of $m^{\mu}(A)$ & -35 & -56 & -126 & -252 & -462 \\
\hline
\end{tabular}

Table 2: Lower and upper bounds for the Möbius transform of a capacity

Observe that the capacities $\mu_{N}^{*}, \mu_{N *}$ are such that their value is 1 on the first lines of the Boolean lattice $2^{N}$, and 0 otherwise. Table 3 (from which Table 2 is obtained) gives the values of $m^{\mu}(N)$ for all capacities $\mu$ on $N$ such that their value on the first $k+1$ lines is 1 , and 0 otherwise. Considering an entry $(n, k)$ of the table, which gives the value of $m^{\mu}(N)$, observe

\begin{tabular}{c|rrrrrrrrrrrr}
$n / k$ & 0 & 1 & 2 & 3 & 4 & 5 & 6 & 7 & 8 & 9 & 10 & 11 \\
\hline$n=1$ & 1 & & & & & & & & & & & \\
$n=2$ & 1 & -1 & & & & & & & & & & \\
$n=3$ & 1 & -2 & 1 & & & & & & & & & \\
$n=4$ & 1 & -3 & 3 & -1 & & & & & & & & \\
$n=5$ & 1 & -4 & 6 & -4 & 1 & & & & & & & \\
$n=6$ & 1 & -5 & 10 & -10 & 5 & -1 & & & & & & \\
$n=7$ & 1 & -6 & 15 & -20 & 15 & -6 & 1 & & & & & \\
$n=8$ & 1 & -7 & 21 & -35 & 35 & -21 & 7 & -1 & & & & \\
$n=9$ & 1 & -8 & 28 & -56 & 70 & -56 & 28 & -8 & 1 & & & \\
$n=10$ & 1 & -9 & 36 & -84 & 126 & -126 & 84 & -36 & 9 & -1 & & \\
$n=11$ & 1 & -10 & 45 & -120 & 210 & -252 & 210 & -120 & 45 & -10 & 1 & \\
$n=12$ & 1 & -11 & 55 & -105 & 330 & -462 & 462 & -330 & 165 & -55 & 11 & -1
\end{tabular}

Table 3: Computation of the upper (red) and lower (blue) bounds of $m^{\mu}(N)$. The value of the capacity $\mu$ is 1 for the $k+1$ first lines of the lattice $2^{N}$. Each entry $(n, k)$ equals $m^{\mu}(N)$

that the value of $m^{\mu}(N \backslash i)$ for any $i \in N$ is given by the entry $(n-1, k-1)$, and so forth: the successive values of $m^{\mu}$ lie on the diagonal $(n, k),(n-1, k-1),(n-2, k-2), \ldots$. Observe that for $n \leq 5$, choosing $k$ which yields the upper bound or the lower bound gives a diagonal passing only through upper or lower bounds. This guarantees that the coordinates of the corresponding capacities in the basis of unanimity games are maximal, which permits to find the diameter. Taking for example $n=5, k=2$ yields the upper bound $\left(\mu_{N}^{*}\right)$ and $k=3$ yields the lower bound $\left(\mu_{N *}\right)$. The coordinates of these capacities are:

\begin{tabular}{|c|ccccc|}
\hline$A$ & $|A|=1$ & $|A|=2$ & $|A|=3$ & $|A|=4$ & $|A|=5$ \\
\hline$m^{\mu_{N}^{*}}(A)$ & 0 & 0 & 1 & -3 & 6 \\
$m^{\mu_{N *}}(A)$ & 0 & 1 & -2 & 3 & -4 \\
\hline
\end{tabular}

Therefore the Euclidean distance between them is (discarding the coordinates for $\varnothing$ and $N$ since the dimension is $2^{n}-2$ )

$$
d\left(m^{\mu_{N}^{*}}, m^{\mu_{N *}}\right)=\sqrt{\left(\begin{array}{l}
5 \\
2
\end{array}\right) \cdot 1+\left(\begin{array}{l}
5 \\
3
\end{array}\right) \cdot 3^{2}+\left(\begin{array}{l}
5 \\
4
\end{array}\right) \cdot 6^{2}}=\sqrt{280} .
$$

This is the diameter for $n=5$ (compare with the diameter in original coordinates $\sqrt{30}$ ). For higher values of $n$, we conjecture that the same method also yields the diameter. 


\section{The polytope of $p$-additive capacities}

The set of $p$-additive capacities is a subpolytope of the polytope of capacities, because the additional restrictions are linear inequalities. For $p=1$, we obtain the polytope of additive capacities, i.e., probability measures. It is easily found that the set of extreme points of this polytope is the set of unanimity games centered on singletons, $u_{\{i\}}, i \in N$. Remark that these are exactly the additive 0-1-capacities.

For $p=2$, the same type of result holds: the vertices of the polytope of 2-additive capacities are simply those vertices of the polytope of capacities which are 2 -additive.

Theorem 3. (Miranda et al. [37, Prop. 11]) The set $\mathcal{C} \leq 2(N)$ of 2 -additive capacities is a $(\kappa(2)-1)$-dimensional polytope, whose extreme points are all at most 2-additive 0-1-capacities. These 0-1-capacities are of three different types:

(i) The unanimity games $u_{\{i\}}, i \in N$ (these are the extreme points of $\mathcal{C}^{1}(N)$ );

(ii) The unanimity games $u_{\{i, j\}},\{i, j\} \subseteq N$;

(iii) The conjugate of the unanimity games $u_{\{i, j\}},\{i, j\} \subseteq N$, given by

$$
\overline{u_{\{i, j,\}}}=u_{i}+u_{j}-u_{\{i, j\}} .
$$

Moreover, the convex decomposition of any $\mu \in \mathfrak{C} \leq 2(N)$ is

$$
\begin{aligned}
\mu= & \sum_{\{i, j\} \subseteq N: m^{\mu}(i j)<0}\left(-m^{\mu}(i j)\right) \overline{u_{\{i, j\}}}+\sum_{\{i, j\} \subseteq N: m^{\mu}(i j)>0} m^{\mu}(i j) u_{\{i, j\}} \\
& +\sum_{i \in N}\left(m^{\mu}(i)+\sum_{j \in N \backslash i: m^{\mu}(i j)<0} m^{\mu}(i j)\right) u_{\{i\}},
\end{aligned}
$$

with the shorthand $i j$ standing for $\{i, j\}$.

The above result permits to know the number of extreme points of $\mathcal{C} \leq 2(N)$. Summing the cardinality of all three types of extreme points, we find $|N|+\left(\begin{array}{c}|N| \\ 2\end{array}\right)+\left(\begin{array}{c}|N| \\ 2\end{array}\right)=|N|^{2}$.

So far, no general result is known for $p>2$. The study becomes very complex in this case because there are extreme points which are not 0-1-valued (Miranda et al. [37]).

\section{The polytopes of $k$-ary capacities and 2-additive $k$-ary capac- ities}

These polytopes have been studied by Grabisch and Labreuche [26]. We denote by $L=$ $\{0,1, \ldots, k\}$ the set of participation levels.

By using a similar proof as in Theorem 1, the following is easy to show.

Theorem 4. The set of extreme points of $\mathcal{C}_{k}(N)$, the polytope of $k$-ary capacities, is the set of 0 -1-valued $k$-ary capacities.

However, unlike the classical case, the following result is more difficult to obtain.

Theorem 5. Let $k \in \mathbb{N}$. The set of extreme points of $\mathcal{C}_{k}^{\leq 2}(N)$, the polytope of 2-additive $k$-ary capacities, is the set of 0 -1-valued 2 -additive $k$-ary capacities. 
Let us define the support of $v$ by:

$$
\operatorname{supp}(v)=\bigcup_{x \in L^{N}: m^{v}(x) \neq 0} \operatorname{supp}(x),
$$

which means that $v$ depends only on the variables in $\operatorname{supp}(v)$.

Theorem 6. For every $k \in \mathbb{N}$, the size of the support of any 0 -1-valued 2-additive $k$-ary capacity is at most 2 .

Comparing with Theorem 3, we see that results that were true for classical 2-additive capacities still hold for $k$-ary capacities, for any $k \in \mathbb{N}$ : vertices are 0-1-valued, and the size of their support is at most 2 .

We give more insight in the extreme points of this polytope. By the above theorem, we know that the size of the support of an extreme point is at most 2 . If the size is 1 , these vertices are simply the unanimity games $u_{x}$ with $x$ of the form $(0, \ldots, 0, \ell, 0, \ldots, 0), \ell \in\{1, \ldots, k\}$. Now, take a vertex, i.e., a 0 -1-valued capacity $v \in \mathcal{C}_{\bar{k}}^{\leq 2}(N)$, with support of size 2 , say $\{1,2\}$. By analogy with the classical case, a profile $x \in L^{N}$ is winning for a 0 -1-valued $v \in \mathcal{C}_{k}^{\leq 2}(N)$ if $v(x)=1$, and it is minimal winning if there is no other winning profile $y$ such that $y \leq x$ coordinatewise. Therefore, minimal winning profiles are antichains in $L^{N}$, and moreover, these antichains are in $L^{\{1,2\}}$. Denumbering vertices amounts therefore to denumbering the number of antichains in $L^{\{i, j\}}$ for any $i \neq j$. This permits to count the total number of vertices of $\mathrm{e}_{k}^{\leq 2}(N)$, which is:

$$
\left[\left(\begin{array}{c}
2 k+2 \\
k+1
\end{array}\right)-2\right] \frac{n(n-1)}{2}-k n(n-2) .
$$

For a given antichain $x^{1}, \ldots, x^{q}$ in $L^{\{1,2\}}$, supposing $x_{1}^{1} \leq \cdots \leq x_{1}^{q}$, the Möbius transform of the corresponding extreme point is easy to obtain: put the value +1 for all elements $x^{1}, \ldots, x^{q},-1$ for all elements $x^{\ell} \vee x^{\ell+1}, \ell=1, \ldots, q-1$ and 0 otherwise (see Figure 2).

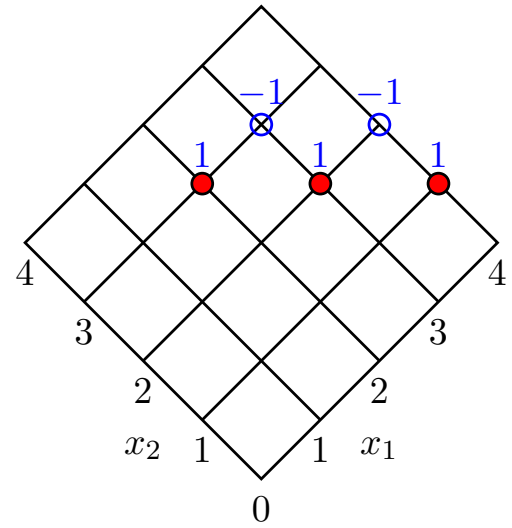

Figure 2: Vertices of $\mathcal{C}_{\vec{k}}^{\leq 2}(N)$ with $k=4$. In red: an antichain in $L^{\{1,2\}}$; values in blue indicate the Möbius transform of the corresponding vertex

\section{The cone of supermodular games}

The set of supermodular games (as well as the set of submodular games) is a cone. A first important result, established by Kuipers et al. [36], is about the set of irredundant inequalities 
defining this cone. Indeed, many inequalities are redundant if (1) is considered for all $S, T \in$ $2^{N}$. In terms of polyhedra, facets correspond to irredundant inequalities, and for the cone of supermodular games, are given by:

$$
v(S \cup\{i, j\})+v(S) \geq v(S \cup i)+v(S \cup j)
$$

for all distinct $i, j \in N$ and all $S \subseteq N \backslash\{i, j\}$.

The cone of supermodular games is not pointed as it contains the set of additive games, which form a linear space. We consider instead the cone of zero-normalized supermodular games, which is pointed because the only zero-normalized additive game is the null game 0 . Let us denote the latter by $\mathcal{G}_{\diamond}(N)$. The problem of finding the extremal rays of $\mathcal{G}_{\diamond}(N)$ is difficult, and was already considered by Shapley in 1971 [47]. He gave in this paper the 37 extremal rays of $\mathcal{G}_{\diamond}(N)$ with $|N|=4$, but no indication for the general case. Later, Rosenmüller and Weidner [43] find all extremal rays of the cone of nonnegative supermodular games (it differs from $\mathcal{G}_{\diamond}(N)$ only by the addition of the extremal rays $\left.u_{\{i\}}, i \in N\right)$ by representing each such game as a maximum over shifted additive games:

$$
v=\max \left(\phi^{1}-\alpha_{1}, \ldots, \phi^{t}-\alpha_{t}\right)
$$

where $\phi^{1}, \ldots, \phi^{t}$ are additive games and $\alpha_{1}, \ldots, \alpha_{t} \in \mathbb{R}_{+}$. Up to some additional conditions, the above representation can be put into a unique canonical form. For a given $v$ and some $H \subseteq\{1, \ldots, t\},|H| \geq 2$, where $t$ pertains to the canonical form, consider the collection $\mathcal{S}_{H}$ of sets such that $v(S)=\phi^{i}(S)-\alpha_{i}$ for every $i \in H$. Then $v$ is an extremal ray if and only if $\phi^{1}, \ldots, \phi^{t}$ are the unique solution of the systems

$$
\phi^{i}(S)-\alpha^{i}=v(S) \quad\left(i \in H, S \in \mathcal{S}_{H}\right)
$$

for all $H \subseteq\{1, \ldots, t\},|H| \geq 2$. We refer the readers to Rosenmüller and Weidner [43] for the details.

More recently, Studený and Kroupa [49] proposed another criterion for finding the extremal rays of $\mathcal{G}_{\diamond}(N)$, based on the fact that supermodular games are exact and their core coincides with the Weber set (see below for definitions).

\section{$7 \quad$ The core of games}

Given a game $v$ on $N$, its core is defined by

$$
\operatorname{core}(v)=\left\{x \in \mathbb{R}^{N} \mid x(S) \geq v(S) \quad \forall S \in 2^{N}, x(N)=v(N)\right\}
$$

where we use the shorthand $x(S)=\sum_{i \in S} x_{i}, S \subseteq N$ (the notation suggests to consider $x$ as an additive game).

The core is an important concept which has been studied in depth, not only in cooperative game theory where it was introduced by Gillies [20] and studied by Shapley [46, 47], but also in decision theory and combinatorial optimization. In game theory, vectors $x \in \operatorname{core}(v)$ are seen as payment vectors. $v(N)$ is the total benefit or cost saving achieved by cooperation of all players in $N$, which has to be shared among the players. Then $x_{i}$ represents the part of the "cake" which is given to player $i$. The condition $x(N)=v(N)$ means that the whole cake is shared (this is called efficiency), while the condition $x(S) \geq v(S)$ means that the part given to coalition $S$ is at least as big as the amount of benefit that $S$ could have realized by itself (this is called coalitional rationality). 
In decision theory, capacities are considered instead, so that the core of a capacity $\mu$ is the set of probability measures dominating $\mu$ (these are also called compatible probability measures). The interpretation is the following: $\mu(A)$ quantifies the amount of certainty of some event $A \subseteq N$. If not enough statistical information is available, $\mu$ is not a probability measure, but represents a lower bound of it. Therefore it makes sense to search for all probability measures which dominates $\mu$. Now, the lower envelope of the core of $\mu$ does not necessarily give $\mu$ back. If this is true, $\mu$ is said to be exact (Schmeidler [45]). The situation described in this paragraph is a particular case of imprecise probabilities; see Walley [52].

In combinatorial optimization, where submodular games are considered, the dual notion of core was introduced by Edmonds [14], namely the base polyhedron associated to a polymatroid $(N, \rho)$ :

$$
B(\rho)=\left\{x \in \mathbb{R}_{+}^{N} \mid x(A) \leq \rho(A) \forall A \in 2^{N}, x(N)=\rho(N)\right\} .
$$

It is easy to see that $B(\rho)=\operatorname{core}(\bar{\rho})$, the core of the conjugate game. Following Edmonds, the properties of the base polyhedron were studied in depth by Fujishige and Tomizawa [19] (see a summary in Fujishige [18, Sec. 3.3]).

\subsection{The core of games on $N$}

The core may be empty: it suffices for example that $\sum_{i \in N} v(\{i\})>v(N)$ to make the core empty. When nonempty, the core is a bounded closed convex polyhedron, whose dimension is at most $n-1$. A necessary and sufficient condition for nonemptiness has been found by Bondareva [5] and Shapley independently [46]. This condition is based on the notion of balanced collections, which are a generalization of partitions. A collection $\mathcal{B}$ of nonempty subsets in $2^{N}$ is balanced if there exist positive coefficients $\lambda_{S}, S \in \mathcal{B}$, such that

$$
\sum_{S \in \mathcal{B}, S \ni i} \lambda_{S}=1 \quad(i \in N) \text {. }
$$

For example, taking $N=\{1,2,3\}, \mathcal{B}=\{\{1,2\},\{1,3\},\{2,3\}\}$ with coefficients $\left(\frac{1}{2}, \frac{1}{2}, \frac{1}{2}\right)$ is a balanced collection. Now, a game $v$ is balanced if for any balanced collection with coefficients $\lambda_{S}, S \in \mathcal{B}$, it holds

$$
\sum_{S \in \mathcal{B}} \lambda_{S} v(S) \leq v(N)
$$

Theorem 7. The core of a game $v$ is nonempty if and only if $v$ is balanced.

The foregoing theorem is usually referenced as the weak form of the Bondareva-Shapley theorem. A sharp form is obtained by imposing inequality (12) only for minimal balanced collections, i.e., those balanced collections which do not contain a proper balanced subcollection.

A complete description of the core is obtained through its extreme points in the case of supermodular games. Let us consider the set of all permutations on $N$, denoted by $\mathfrak{S}(N)$. To any permutation $\sigma \in \mathfrak{S}(N)$, we make correspond a chain in $2^{N}$ :

$$
\varnothing=S_{0} \subset S_{1} \subset \cdots \subset S_{n}=N
$$

with

$$
S_{i}=\{\sigma(1), \ldots, \sigma(i)\} .
$$

Let $v$ be a game. To each permutation $\sigma \in \mathfrak{S}(N)$ we assign a marginal worth vector $x^{\sigma, v}$ in $\mathbb{R}^{N}$ defined by:

$$
\begin{aligned}
x_{\sigma(i)}^{\sigma, v} & :=v\left(S_{i}\right)-v\left(S_{i-1}\right) \\
& =v\left(S_{i-1} \cup \sigma(i)\right)-v\left(S_{i-1}\right) \quad(i \in N) .
\end{aligned}
$$


Observe that

$$
x^{\sigma, v}\left(S_{i}\right)=v\left(S_{i}\right) \quad(i=1, \ldots, n) .
$$

The convex hull of all marginal vectors is called the Weber set:

$$
\operatorname{Web}(v)=\operatorname{conv}\left(\left\{x^{\sigma, v}\right\}_{\sigma \in \mathfrak{S}(N)}\right) .
$$

The following inclusion always holds:

$$
\operatorname{core}(v) \subseteq \operatorname{Web}(v) .
$$

The reverse inclusion holds if and only if $v$ is supermodular. More precisely, we have:

Theorem 8. The following are equivalent.

(i) $v$ is supermodular;

(ii) All marginal vectors $x^{\sigma, v}, \sigma \in \mathfrak{S}(N)$, belong to the core;

(iii) $\operatorname{core}(v)=\operatorname{Web}(v)$;

(iv) $\operatorname{ext}(\operatorname{core}(v))=\left\{x^{\sigma, v}\right\}_{\sigma \in \mathfrak{S}(N)}$.

Parts of this theorem have been shown several times. Up to the knowledge of the author, the earliest version is due to Dempster in 1967 [8]. He proved that the marginal vectors are extreme points of the core for capacities with a nonnegative Möbius transform (usually called belief functions). Shapley showed in 1971 [47] implications (i) $\Rightarrow$ (ii) and (i) $\Rightarrow$ (iv). At the same time, Edmonds proved (i) $\Rightarrow$ (iv) [14], and Ichiishi proved (ii) $\Rightarrow$ (i) in 1981 [35].

Note that so far, no general result is known on the vertices of the core for games which are not supermodular, except on the maximum number of vertices. Derks and Kuipers [12] established that, as for supermodular games, the upper bound of the number of vertices is $n$ !.

Partial results on vertices have been obtained by Núñez and Rafels [39, 40], in particular for almost convex games. A game $v$ on $N$ is almost convex if it satisfies the supermodularity condition (1) for all $S, T \subset N$ (in other words, every subgame $v_{S}$ for $\varnothing \neq S \subset N$ is supermodular). Then, for those games, the set of extreme points of the core is given by

$$
\operatorname{ext}(\operatorname{core}(v))=\bigcup_{i \in N}\left((v(N)-v(N \backslash i))_{i}, \operatorname{ext}\left(\operatorname{core}\left(v^{i}\right)\right)\right) .
$$

We explain the notation. For any $i \in N$, the game $v^{i}$ is a game on $N \backslash i$ defined by

$$
v^{i}(S)=\max (v(S), v(S \cup i)-(v(N)-v(N \backslash i))) \quad(S \subseteq N \backslash i) .
$$

Now, $\left((v(N)-v(N \backslash i))_{i}\right.$, ext $\left.\left(\operatorname{core}\left(v^{i}\right)\right)\right)$ is a $n$-dim vector whose $i$ th component is $v(N)-v(N \backslash i)$, and $n-1$ remaining components are just ext $\left(\operatorname{core}\left(v^{i}\right)\right)$. Núnez and Rafels show that if $v$ is almost convex, then $v^{i}$ too is almost convex, for any $i \in N$. Hence (14) permits to recursively compute the extreme points of the core, coordinate by coordinate.

\subsection{The core of games on set systems}

When the domain of a game is restricted to some family $\mathcal{F} \subset 2^{N}$, the study of the core becomes fairly more complicated. A survey on this topic has been done by the author in [23], to which the reader is referred for details and other results. We give here the most fundamental results, as well as new ones. 
A set system $\mathcal{F}$ is a subcollection of $2^{N}$ containing $N$ and $\varnothing$. We denote by $(v, \mathcal{F})$ a game on $N$ whose domain is $\mathcal{F}$ (this is called a game with restricted cooperation (Faigle [15])). Many examples of set systems have been studied (lattices, matroids, antimatroids, regular set systems, etc., see, e.g., the monograph of Bilbao [1], or [2, 3]). We mainly focus in this section on set systems which are distributive lattices, that is, of the form $\mathcal{F}=\mathcal{O}(N)$ with $N$ endowed with a partial order $\preceq$. Games on such kind of set systems have been introduced by Faigle and Kern [17] under the name game with precedence constraints.

The core of a game with restricted cooperation is defined as in the classical case:

$$
\operatorname{core}(v, \mathcal{F})=\left\{x \in \mathbb{R}^{N} \mid x(S) \geq v(S) \forall S \in \mathcal{F}, x(N)=v(N)\right\} .
$$

While the core is still a convex closed polyhedron of dimension at most $n-1$, unlike the classical case, it may be unbounded, or even not pointed. We study its structure in the next paragraphs. As a general fact, we know from the theory of polyhedra that the core is the Minkovsky sum of its convex part (the convex hull of its vertices) and the conic part, called the recession cone (conic combination of its extremal rays) and denoted by core $(0, \mathcal{F})$, because it is given by

$$
\operatorname{core}(0, \mathcal{F})=\left\{x \in \mathbb{R}^{N} \mid x(S) \geq 0 \forall S \in \mathcal{F}, x(N)=0\right\} .
$$

Nonemptiness. The classical result of Bondareva-Shapley still holds when $2^{N}$ is replaced by $\mathcal{F}$. However, something more can be said.

Theorem 9. (Grabisch and Sudhölter [30]) Let $\mathcal{F}=\mathcal{O}(N, \preceq)$ be a distributive lattice. If $(N, \preceq)$ is connected, the core of any game on $\mathcal{F}$ is nonempty.

Here, "connected" means that the Hasse diagram of the poset, seen as a graph, is connected.

Pointedness. Supposing core $(v, \mathcal{F})$ nonempty, it is pointed if and only if the system

$$
x(S)=0 \quad(S \in \mathcal{F} \backslash\{\varnothing\})
$$

has 0 as unique solution. In this case, we say that $\mathcal{F}$ is nondegenerate, otherwise it is degenerate.

Example 1. Consider $n=3$ and $\mathcal{F}=\{\varnothing, 1,2,123\}$. The recession cone core $(0, \mathcal{F})$ reads

$$
\begin{aligned}
x_{1} & \geq 0 \\
x_{2} & \geq 0 \\
x_{1}+x_{2}+x_{3} & =0 .
\end{aligned}
$$

The core, if nonempty, is pointed because putting equality everywhere leads to a system whose unique solution is 0 . Now, the core is not bounded because the vector $(1,0,-1)$ belongs to $\operatorname{core}(0, \mathcal{F})$.

There are some obvious sufficient conditions for $\mathcal{F}$ to be nondegenerate or degenerate. For example, if $\{i\} \in \mathcal{F}$ for every $i \in N$, or if $\mathcal{F}$ contains a maximal chain of length $n$, then $\mathcal{F}$ is nondegenerate; it is degenerate if there exists a macro-player, that is, a subset $K \subset N$ of at least two elements such that for any $S \in \mathcal{F}$, either $S \supseteq K$ or $S \cap K=\varnothing$. In case of a set system closed under intersection, this turns out to be a sufficient and necessary condition (Faigle et al. $[16])$. 
Boundedness The core is bounded if and only if the conic part is reduced to the zero vector: $\operatorname{core}(0, \mathcal{F})=\{0\}$. The following result, shown by Derks and Reijnierse [13], gives a necessary and sufficient condition for boundedness.

Theorem 10. Let $v$ be a balanced game on a set system $\mathcal{F}$. Then $\operatorname{core}(v, \mathcal{F})$ is bounded if and only if $\mathcal{F}$ is nondegenerate and $\mathcal{F} \backslash\{\varnothing, N\}$ is balanced.

As we will see in the next paragraph, when $\mathcal{F}=\mathcal{O}(N)$, the core is unbounded unless $(N, \preceq)$ is an antichain

Extremal rays of the core. Recall that in a poset $(N, \preceq), i \prec \cdot j$ means $i \prec j$ and there is no $k$ such that $i \prec k \prec j$. The extremal rays of the core when $\mathcal{F}=\mathcal{O}(N)$ have been found by Fujishige and Tomizawa [19], and rediscovered by Derks and Gilles [10].

Theorem 11. Let $\mathcal{F}=\mathcal{O}(N, \preceq)$ be a set system. The conic part of the core reads

$$
\operatorname{core}(0, \mathcal{F})=\operatorname{cone}\left(1_{\{j\}}-1_{\{i\}} \mid i, j \in N \text { such that } j \prec \cdot i\right) \text {. }
$$

The above result says that there is exactly one extremal ray per link in the Hasse diagram of the poset $(N, \preceq)$. Consequently, under the assumption that $\mathcal{F}=\mathcal{O}(N)$, the only case where the core is bounded is when $(N, \preceq)$ is an antichain, i.e., $\mathcal{F}=2^{N}$.

Example 2. Consider $n=4$ and the poset $(N, \preceq)$ given on Figure 1 . Then there are three extremal rays: $(0,0,1,-1),(1,-1,0,0)$ and $(0,-1,1,0)$.

The above result has been recently extended by Faigle et al. [16] for set systems which are closed under intersection.

Theorem 12. Let $(N, \preceq)$ be a poset, and consider a set system $\mathcal{F} \subseteq \mathcal{O}(N, \preceq)$ which is closed under intersection. Then $1_{\{i\}}-1_{\{j\}}$ is a ray of $\operatorname{core}(0, \mathcal{F})$ for every $i \prec \cdot j$ in the poset $(N, \preceq)$, but not necessarily extremal. Moreover, $\operatorname{core}(0, \mathcal{F})=\operatorname{core}(0, \mathcal{O}(N, \preceq))$ if and only if any $S \in$ $\mathcal{O}(N, \preceq) \backslash \mathcal{F}$ can be written as a union of disjoint sets in $\mathcal{F}$.

Example 3. Consider $n=5$ and $\mathcal{F} \subset \mathcal{O}(N)$ depicted on Figure 3 together with $(N, \preceq)$. Observe that $\mathcal{F} \backslash \mathcal{O}(N)=\{1234\}$, and that it is not possible to write 1234 as a union of disjoint

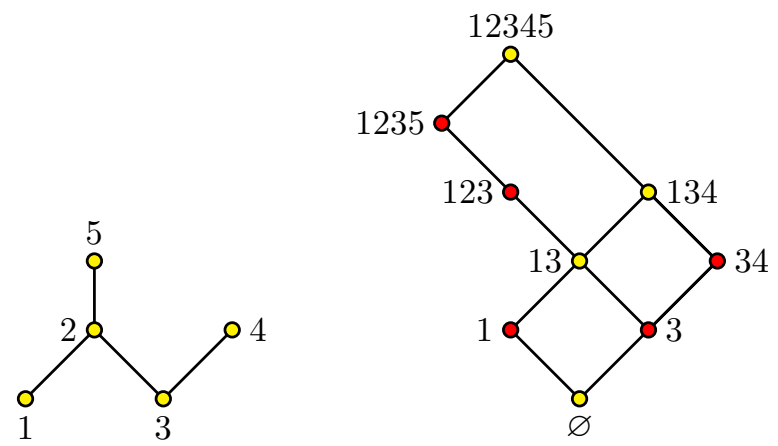

Figure 3: Left: a poset $(N, \preceq)$. Right: a set system $\mathcal{F} \subset \mathcal{O}(N)$. Join-irreducible elements are in red. $\mathcal{O}(N) \backslash \mathcal{F}=\{1234\}$

sets. Hence $\operatorname{core}(0, \mathcal{F}) \neq \operatorname{core}(0, \mathcal{O}(N))$. This can be verified as $r=(0,0,1,-1,0)$, which is extremal in core $(0, \mathcal{O}(N))$, is no more an extremal ray of core $(0, \mathcal{F})$. 
Extreme points of the core. We give now the generalized version of Theorem 8, valid for set systems of the form $\mathcal{F}=\mathcal{O}(N, \preceq)$. Since these set systems are closed under union and intersection, the definition of supermodularity is still valid.

A permutation $\sigma \in \mathfrak{S}(N)$ is compatible with $\mathcal{F}$ if the corresponding maximal chain lies in $\mathcal{F}$. We denote by $\mathfrak{S}(\mathcal{F})$ the set of permutations compatible with $\mathcal{F}$.

Theorem 13. Let $v$ be a game on $(N, \mathcal{F})$ with $\mathcal{F}=\mathcal{O}(N, \preceq)$. The following propositions are equivalent.

(i) $v$ is supermodular;

(ii) $x^{\sigma, v} \in \operatorname{core}(v, \mathcal{F})$ for all $\sigma \in \mathfrak{S}(\mathcal{F})$;

(iii) $\operatorname{core}(v, \mathcal{F})=\operatorname{conv}\left(\left\{x^{\sigma, v} \mid \sigma \in \mathfrak{S}(\mathcal{F})\right\}\right)+\operatorname{core}(0, \mathcal{F})$;

(iv) $\operatorname{ext}(\operatorname{core}(v, \mathcal{F}))=\left\{x^{\sigma, v} \mid \sigma \in \mathfrak{S}(\mathcal{F})\right\}$.

As in the classical case, this result has been shown both in combinatorial optimization, by Fujishige and Tomizawa [19], and in game theory, by Derks and Gilles [10].

Bounded faces of the core. We consider in this section the case where $\mathcal{F}$ is of the form $\mathcal{O}(N, \preceq)$. The core being unbounded unless $(N, \preceq)$ is an antichain, it is important to search for remarkable bounded subsets, for example bounded faces. These are obtained through the notion of normal collection (Grabisch [22]). It is well known from the theory of polyhedra that faces of a polyhedron defined by a system of inequalities are obtained by turning some of the inequalities into equalities. Since in the case of the core, inequalities correspond to subsets of $N$, a normal collection is a collection of sets whose corresponding face is bounded. Formally, a normal collection is any collection $\mathcal{N} \subset \mathcal{F} \backslash\{\varnothing, N\}$ of sets such that

$$
\operatorname{core}_{\mathcal{N}}(0, \mathcal{F})=\left\{x \in \mathbb{R}^{N} \mid x(S) \geq 0 \forall S \in \mathcal{F}, x(S)=0 \forall S \in \mathcal{N} \text {, and } x(N)=0\right\}=\{0\} .
$$

Note that by convention, $N \notin \mathcal{N}$. If $\operatorname{core}(v, \mathcal{F}) \neq \varnothing$, $\operatorname{core}_{\mathcal{N}}(v)$ is a bounded face of the core. It may be empty even if $\operatorname{core}(v, \mathcal{F})$ is nonempty. The union of all bounded faces of $\operatorname{core}(v, \mathcal{F})$ is called the bounded core (Grabisch and Sudhölter [30]), and denoted by $\operatorname{core}^{b}(v, \mathcal{F})$. It is equivalently defined by

$$
\operatorname{core}^{b}(v, \mathcal{F})=\left\{x \in \operatorname{core}(v, \mathcal{F}) \mid \forall j \prec \cdot i, \forall \epsilon>0, x+\epsilon\left(1_{\{i\}}-1_{\{j\}}\right) \notin \operatorname{core}(v, \mathcal{F})\right\}
$$

and was axiomatized as a solution concept in [30].

We give a very simple criterion to find normal collections. We say that an extremal ray $r$ of core $(0, \mathcal{F})$ is deleted by equality $x(S)=0, S \in \mathcal{F}$, if $\operatorname{core}_{\{S\}}(0, \mathcal{F})=\{x \in \operatorname{core}(0, \mathcal{F}) \mid x(S)=0\}$ does not contain $r$ any more.

Lemma 1. Let $\mathcal{F}=\mathcal{O}(N)$. For $i, j \in N$ such that $j \prec i$, the extremal ray $1_{\{j\}}-1_{\{i\}}$ is deleted by equality $x(S)=0$ if and only if $S \ni j$ and $S \not \nexists i$.

Example 4. (Ex. 3 continued) The three extremal rays can be deleted simply by the subset $\{1,3\}$. Hence $\mathcal{N}=\{\{1,3\}\}$ is a normal collection, but $\mathcal{N}=\{\{1\},\{3\}\}$ and $\mathcal{N}=\{\{1\},\{3\},\{1,3\}\}$ are normal collections as well.

The above simple example shows that in general there are many normal collections, inducing faces of various dimensions. Obviously, the most interesting ones are those of largest dimension, i.e., those defined by the smallest normal collections (in cardinality). It is proved in (Grabisch 
[22]) that a normal collection contains at least $h(N)$ subsets, where $h(N)$ is the height of $(N, \preceq)$, i.e., the length of a longest maximal chain. Observe that in Example $4, h(N)=1$. It is useful to introduce the following properties of normal collections. Consider $(N, \preceq)$ with $h(N)>0$, and a normal collection $\mathcal{N}=\left\{N_{1}, \ldots, N_{q}\right\}$.

(i) $\mathcal{N}$ is a minimal collection if no proper subcollection is normal.

(ii) $\mathcal{N}$ is a nested collection if it is a chain in $\mathcal{F}$.

It turns out that for supermodular games, minimal nested normal collections are sufficient to generate all bounded faces of maximal dimension. We denote by $\mathcal{M N \mathcal { N C }}(\mathcal{F})$ the set of minimal nested normal collections of $\mathcal{F}$.

Theorem 14. (Grabisch and Sudhölter [31])

(i) For any supermodular game $v$ and any nested normal collection $\mathcal{N}$ of $\mathcal{F}$, $\operatorname{core}_{\mathcal{N}}(v, \mathcal{F}) \neq \varnothing$. Moreover, if $v$ is strictly supermodular, then $\operatorname{dim} \operatorname{core}_{\mathcal{N}}(v, \mathcal{F})=n-|\mathcal{N}|-1$.

(ii) For any supermodular game $v$,

$$
\operatorname{core}^{b}(v, \mathcal{F})=\bigcup_{\mathcal{N} \in \mathcal{M} \mathcal{N} \mathcal{N C}(\mathcal{F})} \operatorname{core}_{\mathcal{N}}(v, \mathcal{F})
$$

Moreover, no term in the union is redundant if $v$ is strictly supermodular.

(iii) Let $\mathcal{N}$ be a normal collection of $\mathcal{F}$. If $v$ is strictly supermodular, then $\operatorname{core}_{\mathcal{N}}(v, \mathcal{F}) \neq \varnothing$ if and only if $\mathcal{N}$ is nested.

\section{The $k$-additive core of games}

Through the concept of $k$-additive games, there is a natural generalization of the core. Indeed, using the identity between a vector $x \in \mathbb{R}^{N}$ and an additive game on $N$, the core of a game $v$ can be defined as the set of additive games $\phi$ such that $\phi \geq v$ pointwise and $\phi(N)=v(N)$. Since additive games are 1-additive games, it is natural to introduce the $k$-additive core as the set of (at most) $k$-additive games dominating a game $v$ and coinciding on $N$ :

$$
\operatorname{core}^{k}(v)=\left\{\phi \in \mathcal{G}^{\leq k}(N) \mid \phi(S) \geq v(S), \forall S \in 2^{N}, \phi(N)=v(N)\right\} .
$$

The $k$-additive core has been proposed by Vassil'ev [51] and rediscovered later by Grabisch and Miranda [28, 38]. The main advantage of the $k$-additive core is that, contrarily to the core, it is never empty as soon as $k \geq 2$. However, it is unbounded.

Theorem 15. (Miranda and Grabich [38]) The $k$-additive core is a convex polyhedron, nonempty for every game as soon as $k \geq 2$.

Theorem 16. (Gonzalez and Grabisch [21]) For all $k \geq 2$, the $k$-additive core is unbounded and pointed. Moreover, for $k=2$, its extremal rays are of the form $1_{i}-1_{i j}$, for all $i \neq j \in N$. 


\subsection{The $k$-additive core as a solution concept in cooperative game theory}

Though its interpretation in cooperative game theory is not immediately clear, it can still be explained in terms of payoff vectors, thus giving a new concept of solution of a game. Indeed, any $\phi \in \operatorname{core}^{k}(v)$ can be written as, using (7) and recalling that $\phi$ is $k$-additive:

$$
\phi(S)=\sum_{\substack{T \subseteq S \\|T| \leq k}} m^{\phi}(T) \quad\left(S \in 2^{N}\right) .
$$

Now, $m^{\phi}$ can be interpreted as a general payoff vector, in the sense that a payoff is given not only to individuals, but also to coalitions, up to size $k$. Payments may be negative, but the total sum allocated to a coalition $S$ is always at least as large as $v(S)$. If a payment only to individuals is eventually required, then a second step is necessary, consisting in sharing the payment given to coalitions among their individuals. This can be done by different means, e.g., sharing functions, bargaining, or when the payment is negative, by using rules for bankruptcy problems. Let us focus only on sharing functions.

A sharing function is a mapping $q: 2^{N} \backslash\{\varnothing\} \times N \rightarrow[0,1]$ satisfying

$$
q(K, i)=0 \text { if } i \notin K, \sum_{i \in K} q(K, i)=1, \quad \varnothing \neq K \subseteq N .
$$

We denote by $\mathcal{Q}(N)$ the set of all sharing functions. Given $\phi \in \operatorname{core}^{k}(v)$ and a sharing function $q, x^{q}(\phi) \in \mathbb{R}^{N}$ is an efficient payoff vector defined by

$$
x_{i}^{q}(\phi)=\sum_{S \ni i} q(S, i) m^{\phi}(S) \quad(i \in N) .
$$

Now, the set of all such payoff vectors, considering all possible sharing functions, is the selectope of $\phi$ (Derks, Haller and Peters [11]):

$$
\operatorname{sel}(\phi)=\left\{x^{q}(\phi) \mid q \in \mathcal{Q}(N)\right\}
$$

A surprising result shown by Grabisch and $\mathrm{Li}$ [27] is the following: if the sharing function gives, for every $K$, a nonnull portion of $m^{\phi}(K)$ to every $i \in K$, then the selectope of $\phi$ is the set of all efficient payoff vectors. Formally:

Theorem 17. Let $2 \leq k \leq n$. For any $q \in \mathcal{Q}(N)$ such that $q(K, i)>0$ for all $\varnothing \neq K \subseteq N$ and $i \in K$, for any game $v$, we have

$$
\left\{x^{q}(\phi) \mid \phi \in \operatorname{core}^{k}(v)\right\}=\left\{x \in \mathbb{R}^{N} \mid x(N)=v(N)\right\} .
$$

This result shows that the $k$-additive core is a very large set, and some selection has to be made in it. This problem has been addressed by Gonzalez and Grabisch [21]. They propose to take those elements in $\operatorname{core}^{k}(v)$ which minimize the total amount of payment to non-singleton coalitions.

\subsection{Vertices of the $k$-additive core}

A result similar to Theorem 8 has been obtained for the $k$-additive core, giving extreme points of it for a certain class of games. However, the study of the extreme points is much more complex than for the classical case, and not all vertices are known so far. We present below the machinery to find some of them, details can be found in (Grabisch and Miranda [28]). 
We begin by introducing some notation and new notions. We denote by $\mathcal{P}^{k}(N)$ the collection of subsets of $N$ containing at most $k$ elements, and we use also $\mathcal{P}_{*}^{k}(N)=\mathcal{P}^{k}(N) \backslash\{\varnothing\}$. Given $\ell \geq 2$, a game $v$ is said to be $\ell$-monotone if for any family $A_{1}, \ldots, A_{\ell}$ of sets in $2^{N}$, it holds:

$$
v\left(\bigcup_{i=1}^{\ell} A_{i}\right) \geq \sum_{\substack{K \subseteq\{1, \ldots, \ell\} \\ K \neq \varnothing}}(-1)^{|K|+1} v\left(\bigcap_{j \in K} A_{j}\right) .
$$

Observe that 2-monotonicity is nothing but supermodularity. A game is totally monotone if it is $\ell$-monotone for every $\ell \geq 2$. Totally monotone capacities are called belief functions. It is well known that $v$ is totally monotone if and only if $m^{v}(S) \geq 0$ for all $S \subseteq N,|S|>1$.

We consider the subset of the $k$-additive core formed by all core elements which are totally monotone:

$$
\operatorname{core}_{\infty}^{k}(v)=\left\{\phi \in \operatorname{core}^{k}(v)\left|m^{\phi}(S) \geq 0, \forall S \in 2^{N},\right| S \mid>1\right\} .
$$

In order to find vertices, the main idea is to find a substitute of marginal vectors, hence of permutations on $N$. Since now the payment is done to every coalition of size at most $k$ instead to individuals, permutations ahould be defined on $\mathcal{P}_{*}^{k}(N)$ instead of $N$. Let us then consider a given total order $\prec$ on $\mathcal{P}_{*}^{k}(N)$. For any $B \in \mathcal{P}_{*}^{k}(N)$, we define the achievable family of $B$ induced by $\prec$ by

$$
\mathcal{A}(B):=\left\{A \subseteq N \mid A \supseteq B, \forall K \subseteq A, K \in \mathcal{P}_{*}^{k}(N), K \preceq B\right\} .
$$

Example 5. Let us take $n=3, k=2$, and the total order $1 \prec 2 \prec 12 \prec 13 \prec 23 \prec 3$ on $\mathcal{P}_{*}^{2}(N)$. The achievable families are given on Figure 4 in red. Observe that $\mathcal{A}(\{3\})$ is a Boolean lattice, and that $\mathcal{A}(13)=\varnothing$.

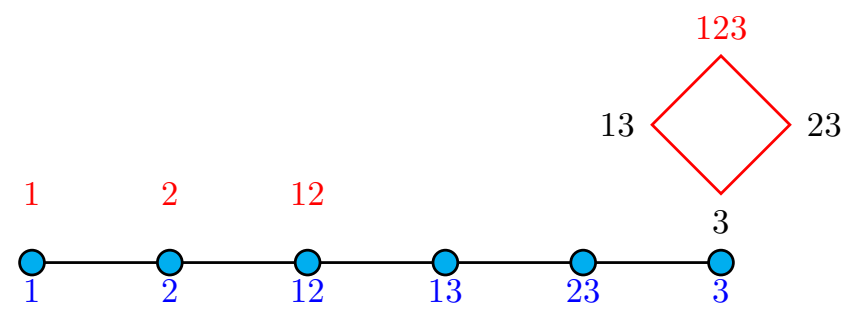

Figure 4: A total order (in blue) on $\mathcal{P}_{*}^{2}(N)$ with $n=3$ and the induced achievable families (in red)

It is easy to see that if $|B|=1$, then $\mathcal{A}(B) \neq \varnothing$ because $B \in \mathcal{A}(B)$ in this case. Also, $\{\mathcal{A}(B)\}_{B \in \mathcal{P}_{*}^{k}(N)}$ is a partition of $\mathcal{P}(N) \backslash\{\varnothing\}$.

We consider now orders with peculiar properties. A total order $\prec$ is said to be

(i) compatible if for all $i, j \in N, i \prec j$ implies $S \cup i \prec S \cup j$, for any $S \in \mathcal{P}^{k-1}(N), i, j \notin S$;

(ii) $\subseteq$-compatible if $A \subseteq B$ implies $A \prec B$;

(iii) strongly compatible if it is compatible and $\subseteq$-compatible;

(iv) weakly compatible if it is compatible but not $\subseteq$-compatible. 
Observe that in Ex. 5, the order is compatible but not strongly compatible. It can be shown that if $\prec$ is compatible, then $\mathcal{A}(B)$ is either empty or an interval $[B, \tilde{B}]$, that is, a Boolean lattice. Moreover, $\mathcal{A}(B)$ is nonempty for all $B \in \mathcal{P}_{*}^{k}(N)$ if and only if $\prec$ is $\subseteq$-compatible. It follows that for strongly compatible orders, all achievable families are nonempty Boolean lattices.

Achievable families play the rôle of maximal chains. As it can be easily checked, with $k=1$, total orders on singletons are permutations on $N$, and all these orders are strongly compatible. Moreover, taking a permutation $\sigma$ on $N$ and considering the order $\sigma(1) \prec \cdots \prec \sigma(n)$, we get

$$
\mathcal{A}(\{\sigma(j)\})=[\{\sigma(j)\},\{\sigma(1), \ldots, \sigma(j)\}],
$$

i.e., the top element of the lattice is $\{\sigma(1), \ldots, \sigma(j)\}$. Then the collection of all top elements gives the maximal chain associated to $\sigma$ (see Example 6 below).

The next step is to define the counterpart of marginal vectors. Consider a game $v$ on $N$, and a total order $\prec$ on $\mathcal{P}_{*}^{k}(N)$. The game $v_{\prec}$ is defined through its Möbius transform $m_{\prec}$ by

$$
m_{\prec}(B):= \begin{cases}\sum_{A \in \mathcal{A}(B)} m(A), & \text { if } \mathcal{A}(B) \neq \varnothing \\ 0, & \text { otherwise }\end{cases}
$$

for all $B \in \mathcal{P}_{*}^{k}(N)$, and $m_{\prec}(\varnothing):=0$.

Example 6. We consider $n=3, k=1$ and the order on singletons $2 \prec 1 \prec 3$. The achievable families as well as the corresponding maximal chain is given in Figure 5 . Computing $m_{\prec}$ by
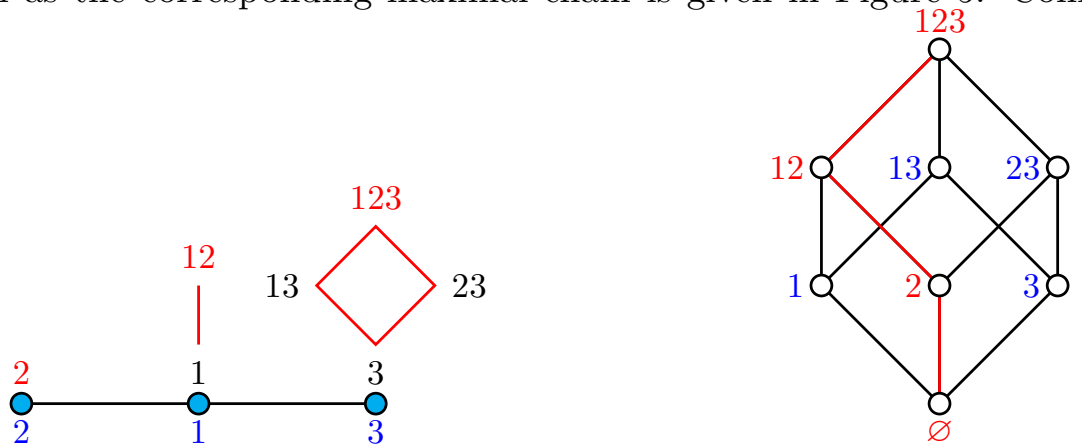

Figure 5: Left: achievable families for the order $2 \prec 1 \prec 3$. Right: corresponding maximal chain in $\left(2^{N}, \subseteq\right)$

(15) yields:

$$
\begin{aligned}
& m_{\prec}(2)=m(2)=v(2)=x_{2}^{\sigma, v} \\
& m_{\prec}(1)=m(1)+m(12)=v(12)-v(2)=x_{1}^{\sigma, v} \\
& m_{\prec}(3)=m(3)+m(13)+m(23)+m(123)=v(123)-v(12)=x_{3}^{\sigma, v},
\end{aligned}
$$

hence the marginal vector $x^{\sigma, v}$ is recovered.

Elementary properties of $v_{\prec}$ are:

(i) If $\prec$ is compatible and $\mathcal{A}(B)$ is nonempty, then $v_{\prec}(\check{B})=v(\check{B})$;

(ii) If $\prec$ is compatible and $v$ is $k$-monotone, then $v_{\prec}$ is infinitely monotone.

Observe that the first property is similar to (13). Recalling that supermodularity is 2-monotonicity, the following result is a generalization of the Shapley-Ichiishi result. 
Theorem 18. A game $v$ is $(k+1)$-monotone if and only if for all compatible orders $\prec, v_{\prec}$ is an element of the $k$-additive core. Moreover, when $v$ is $(k+1)$-monotone,

(i) If $\prec$ is strongly compatible, then $v_{\prec}$ is a vertex of $\operatorname{core}^{k}(v)$;

(ii) If $\prec$ is compatible, then $v_{\prec}$ is a vertex of $\operatorname{core}_{\infty}^{k}(v)$.

However, unlike the classical case $k=1$, the above construction based on strongly compatible orders is far from giving all vertices. In fact, the number of vertices obtained by this construction is at most $\frac{n !}{k !}$ (observe that with $k=1$, we recover that the maximum number of vertices of the core is $n$ !). When $k=n-1$, it is however possible to get all vertices, as shown in the next theorem.

Theorem 19. Let $v$ be any game in $\mathcal{G}(N)$, with Möbius transform $m^{v}$.

(i) If $m^{v}(N)>0, \operatorname{core}^{n-1}(v)$ contains exactly $2^{n-1}$ (if $n$ is even) or $2^{n-1}-1$ (if $n$ is odd) vertices, among which $n$ vertices come from strongly compatible orders. They are given by their Möbius transform:

$$
m_{B_{0}}^{*}(K)= \begin{cases}m^{v}(K), & \text { if } K \nsupseteq B_{0} \\ m^{v}(K)+(-1)^{\left|K \backslash B_{0}\right|} m^{v}(N), & \text { else }\end{cases}
$$

for all $B_{0} \subset N$ such that $\left|N \backslash B_{0}\right|$ is odd.

(ii) If $m^{v}(N)=0$, then there is only one vertex, which is $v$ itself.

(iii) If $m^{v}(N)<0, \operatorname{core}^{n-1}(v)$ contains exactly $2^{n-1}-1$ (if $n$ is odd) or $2^{n-1}-2$ (if $n$ is even) vertices, of which none comes from a strongly compatible order. They are given by their Möbius transform:

$$
m_{B_{0}}^{*}(K)= \begin{cases}m^{v}(K), & \text { if } K \nsupseteq B_{0} \\ m^{v}(K)-(-1)^{\left|K \backslash B_{0}\right|} m^{v}(N), & \text { else }\end{cases}
$$

for all $B_{0} \subset N$ such that $\left|N \backslash B_{0}\right|$ is even.

\section{References}

[1] J. M. Bilbao. Cooperative games on combinatorial structures. Kluwer Academic Publishers, Boston, 2000.

[2] J. M. Bilbao. Cooperative games under augmenting systems. SIAM J. Discrete Math., $17: 122-133,2003$.

[3] J. M. Bilbao, E. Lebrón, and N. Jiménez. The core of games on convex geometries. European Journal of Operational Research, 119:365-372, 1999.

[4] G. Birkhoff. On the combination of subalgebras. Proc. Camb. Phil. Soc., 29:441-464, 1933.

[5] O. Bondareva. Some applications of linear programming to the theory of cooperative games. Problemy Kibernetiki, 10:119-139, 1963. in Russian.

[6] G. Choquet. Theory of capacities. Annales de l'Institut Fourier, 5:131-295, 1953.

[7] Y. Crama and P. Hammer. Boolean functions. Number 142 in Encyclopedia of Mathematics and Its Applications. Cambridge University Press, 2011. 
[8] A. P. Dempster. Upper and lower probabilities induced by a multivalued mapping. Ann. Math. Statist., 38:325-339, 1967.

[9] D. Denneberg. Non-Additive Measure and Integral. Kluwer Academic, 1994.

[10] J. Derks and R. Gilles. Hierarchical organization structures and constraints on coalition formation. Int. J. of Game Theory, 24:147-163, 1995.

[11] J. Derks, H. Haller, and H. Peters. The selectope for cooperative games. Int. J. of Game Theory, 29:23-38, 2000.

[12] J. Derks and J. Kuipers. On the number of extreme points of the core of a transferable utility game. In P. Borm and H. Peters, editors, Chapters in Game Theory (in honor of Stef Tijs), pages 83-97. Kluwer Academic, 2002.

[13] J.J.M. Derks and H. Reijnierse. On the core of a collection of coalitions. Int. J. of Game Theory, 27:451-459, 1998.

[14] J. Edmonds. Submodular functions, matroids, and certain polyhedra. In R. Guy, H. Hanani, N. Sauer, and J. Schönheim, editors, Proc. of the Calgary Int. Conf. on Combinatorial Structures and Their Applications, pages 66-87, 1970.

[15] U. Faigle. Cores of games with restricted cooperation. ZOR - Methods and Models of Operations Research, 33:405-422, 1989.

[16] U. Faigle, M. Grabisch, A. Jiménez-Losada, and M. Ordóñez. Games on concepts lattices: Shapley value and the core. Discrete Applied Mathematics, 198:29-47, 2016.

[17] U. Faigle and W. Kern. The Shapley value for cooperative games under precedence constraints. Int. J. of Game Theory, 21:249-266, 1992.

[18] S. Fujishige. Submodular functions and optimization, volume 58 of Annals of Discrete Mathematics. Elsevier, Amsterdam, 2nd edition, 2005.

[19] S. Fujishige and N. Tomizawa. A note on submodular functions on distributive lattices. $J$. of the Operations Research Society of Japan, 26:309-318, 1983.

[20] D. Gillies. Some theorems on n-person games. PhD thesis, Princeton, New Jersey, 1953.

[21] S. Gonzalez and M. Grabisch. Preserving coalitional rationality for non-balanced games. Int. J. of Game Theory, 44:733-760, 2015.

[22] M. Grabisch. Ensuring the boundedness of the core of games with restricted cooperation. Annals of Operations Research, 191:137-154, 2011.

[23] M. Grabisch. The core of games on ordered structures and graphs. Annals of Operations Research, 204:33-64, 2013. doi: 10.1007/s10479-012-1265-4.

[24] M. Grabisch. Set Functions, Games and Capacities in Decision Making, volume 46 of Theory and Decision Library C. Springer, 2016.

[25] M. Grabisch and Ch. Labreuche. Capacities on lattices and $k$-ary capacities. In $3 d$ Int, Conf. of the European Soc. for Fuzzy Logic and Technology (EUSFLAT 2003), pages 304307, Zittau, Germany, September 2003. 
[26] M. Grabisch and Ch. Labreuche. On the decomposition of Generalized Additive Independence models. arXiv:1601.05978, 2016.

[27] M. Grabisch and T. Li. On the set of imputations induced by the $k$-additive core. Eur. J. of Operational Research, 214:697-702, 2011.

[28] M. Grabisch and P. Miranda. On the vertices of the $k$-additive core. Discrete Mathematics, 308:5204-5217, 2008. doi:10.1016/j.disc.2007.09.042.

[29] M. Grabisch and P. Miranda. Exact bounds of the Möbius inverse of monotone set functions. Discrete Applied Mathematics, 186:7-12, 2015.

[30] M. Grabisch and P. Sudhölter. The bounded core for games with precedence constraints. Annals of Operations Research, 201:251-264, 2012. doi: 10.1007/s10479-012-1228-9.

[31] M. Grabisch and P. Sudhölter. On the restricted cores and the bounded core of games on distributive lattices. Eur. J. of Operational Research, 235:709-717, 2014 . doi: 10.1016/j.ejor.2013.10.027.

[32] P. L. Hammer and S. Rudeanu. Boolean Methods in Operations Research and Related Areas. Springer, 1968.

[33] J. C. Harsanyi. A simplified bargaining model for the $n$-person cooperative game. International Economic Review, 4:194-220, 1963.

[34] C. R. Hsiao and T. E. S. Raghavan. Multichoice cooperative games. In B. Dutta, editor, Proc. of the Int. Conf. on Game Theory and Economic Applications, New Delhi, India, 1990.

[35] T. Ichiishi. Super-modularity: applications to convex games and to the greedy algorithm for LP. J. Econom. Theory, 25:283-286, 1981.

[36] J. Kuipers, D. Vermeulen, and M. Voorneveld. A generalization of the Shapley-Ichiishi result. Int. J. of Game Theory, 39:585-602, 2010.

[37] P. Miranda, E. Combarro, and P. Gil. Extreme points of some families of non-additive measures. Eur. J. of Operational Research, 174:1865-1884, 2006.

[38] P. Miranda and M. Grabisch. k-balanced games and capacities. Eur. J. of Operational Research, 200:465-472, 2010.

[39] M. Núñez and C. Rafels. On extreme points of the core and reduced games. Annals of Operations Research, 84:121-133, 1998.

[40] M. Núñez and C. Rafels. Characterization of the extreme core allocations of the assignment game. Games and Economic Behavior, 44:311-331, 2003.

[41] R. O'Donnell. Analysis of Boolean functions. Cambridge University Press, 2014.

[42] D. Radojevic. The logical representation of the discrete Choquet integral. Belgian Journal of Operations Research, Statistics and Computer Science, 38:67-89, 1998.

[43] J. Rosenmüller and H. G. Weidner. Extreme convex set functions with finite carrier: general theory. Discrete Mathematics, 10:343-382, 1974. 
[44] G. C. Rota. On the foundations of combinatorial theory I. Theory of Möbius functions. Zeitschrift für Wahrscheinlichkeitstheorie und Verwandte Gebiete, 2:340-368, 1964.

[45] D. Schmeidler. Cores of exact games I. J. Math. Analysis and Appl., 40:214-225, 1972.

[46] L. S. Shapley. On balanced sets and cores. Naval research Logistics Quarterly, 14:453-460, 1967.

[47] L. S. Shapley. Cores of convex games. Int. J. Game Theory, 1:11-26, 1971.

[48] R. Stanley. Two poset polytopes. Discrete and Computational Geometry, 1:9-23, 1986.

[49] M. Studený and T. Kroupa. Core-based criterion for extreme supermodular functions. arXiv:1410.8395v1, 2014.

[50] M. Sugeno. Theory of fuzzy integrals and its applications. PhD thesis, Tokyo Institute of Technology, 1974.

[51] V. Vassil'ev. Polynomial cores of cooperative games. Optimizacia, 21:5-29, 1978. in russian.

[52] P. Walley. Coherent lower (and upper) probabilities. Technical Report 22, University of Warvick, Coventry, 1981. 